\section{Genetic Assessment of Watermelon Germplasm using the AFLP Technique}

\author{
Ke-peng Che, Chun-yang Liang, Yue-guang Wang, De-min Jin, and \\ Bin Wang ${ }^{1}$ \\ Institute of Genetics, Chinese Academy of Sciences, Beijing, China
}

\author{
Yong Xu, Guo-bing Kang, and Hai-ying Zhang \\ National Engineering Research Center for Vegetables, Beijing, China
}

Additional index words. AFLP, cluster analysis, genetic diversity, watermelon, germplasm identification

\begin{abstract}
Amplified fragment length polymorphism (AFLP) analyses were used to assess genetic diversity among 30 genotypes of watermelon [Citrullus lanatus (Thunb.) Mansf.] representing a broad genetic base, including breeding lines and commercial germplasm. Eight AFLPprimer combinations selected from 64 primer combinations were polymorphic. The polymorphism was $13.0 \%$ to $31.9 \%$ within the 28 cultivars examined, and $45.3 \%$ to $64.2 \%$ among all the genotypes. Each genotype could be successfully distinguished based on AFLP scoring. Cluster grouping of accessions based on the AFLP analysis was consistent with that from classification by pedigrees and ecotypes.
\end{abstract}

The assessment of germplasm diversity is a prerequisite for effective utilization of genetic resources. Watermelon is a commonly grown species worldwide that includes a diverse assay of cultivated germplasm and wild types (Bailey, 1930; Bates and Robinson, 1995; Jeffrey, 1975). New cultivars, especially hybrids, are being developed continuously. Traditional taxonomy of $C$. lanatus is mainly based on unique morphological characters, such as fruit shape, rind color, seedcoat, shape, and disease and insect resistance traits. However, range of morphological characters is relatively small and not sufficient to differentiate some watermelon genotypes. Additionally, many of these traits are developmentally regulated or influenced by environmental conditions (Provvidenti, 1994; Taylor, 1989).

Enzyme and seed protein polymorphism has been used to estimate the genetic diversity of watermelon germplasm (Biles et al., 1989; Navot and Zamir, 1987; Walters et al., 1991; Zamir et al., 1984). Polymorphic differences were observed between cultivated and wild genotypes. The polymorphism within cultivars is smaller than that between cultivars and wild genotypes. The effectiveness of enzyme systems for genetic assessment of watermelon, however, is limited by the number of systems available and the paucity of isozymic variation.

Compared with protein markers, more efficient and reliable DNA-based markers, such as random amplified polymorphic DNA (RAPD) and simple sequence repeat (SSR) markers, have been used for assessing diversity in watermelon (Hashizume et al., 1993; Jarret et al., 1997; Lee et al., 1996; Zhang et al., 1994). However, not all of the cultivars analyzed by RAPD markers were discriminated

Received for publication 28 Sept. 2001. Accepted for publication 7 Mar. 2002.

${ }^{1}$ To whom reprint requests should be addressed. E-mail address: bwang@genetics.ac.cn
(Hashizume et al., 1993; Lee et al., 1996; Levi et al., 2001; Zhang et al., 1994). Although SSR markers were comparatively more effective in detecting genetic polymorphism between cultivated and wild types of watermelon, the number of markers is relatively limited (Jarret et al., 1997).

A comparative analysis of the performance of several types of molecular markers (i.e., AFLP, RFLP, SSR, and RAPD) for genetic diversity measurement has been carried out in several plant species (Garcia-Mas et al., 2000; Li et al., 2000; Lin et al., 1996; Pejic et al., 1998; Russell et al., 1997; Sharma et al., 1996; Wang et al., 1999). Results indicate that AFLP markers are more reliable and polymorphic than other markers examined. However, to our knowledge, the AFLP technique has not been used for genetic assessment of watermelon germplasm.

The objective of this research was to use the AFLP method to detect diversity and determine genetic relationships among different watermelon genotypes. The investigation will provide essential information for future marker-facilitated breeding of watermelon.

\section{Materials and Methods}

Germplasm. Thirty watermelon genotypes representing a wide range of breeding and commercially available germplasm with different origins were used in the study. Five commercial $F_{1}$ hybrids (No. 3, 7, 10, 13, 16 in Table 1), eight parents of $F_{1}$ hybrid cultivars (No. 1, 2, $5,6,8,9,14,15$ in Table 1), 15 experimental hybrids (No. 4, 11, 12, 17, 18, 19, 20, 21, 22, $23,24,25,26,27,28$ in Table 1), and two wild genotypes (No. 29, 30 in Table 1) were examined. Genotypes $1-28$ belong to the cultivated botanical variety $C$. lanatus var. lanatus, while accession 29 is wild Tsamma melon (C. lanatus var. citroides), and accession 30 is Egusi melon (C. lanatus var. lanatus/egusi). These genotypes are described in Table 1. Genotypes
11, 17, and 19 are tretraploid; the others are diploid.

The $F_{1}$ hybrids were provided by the Pomological Institute of the Chinese Academy of Agricultural Science, Zhengzhou City, China; and Northwest Agricultural University, Yangling City, China. Wild genotypes and American cultivars were obtained from R. Provvidenti of Cornell Univ., Ithaca, N.Y. The remaining accessions were provided by the National Engineering Research Center for Vegetables, Beijing, China.

DNA extraction and AFLP analysis. Total genomic DNA was isolated from young leaves using a modified CTAB method based on Murry (1980). AFLP analysis was carried out using the Gibco-BRL AFLP Kit protocol (Cat. No. 10719-011, Life Technologies Inc.). DNA (500 ng) was double-digested with Eco RI and Mse I, and the digested DNA fragments were ligated with Eco RI and Mse I adaptors, respectively. A preselective amplification was carried out with Eco RI+A and Mse I+C primers, and the PCR product was diluted in a ratio of 1:15 with TE buffer, and then used as a template for the selective amplification. Selective amplification primers $(E c o \mathrm{RI}+2 /+3)$ were radiolabeled with $\left(r-{ }^{33} \mathrm{p}\right)$ ATP (purchased fromAmersham Bioscience Inc.). The products of AFLP analysis were separated on 6\% PAGE sequencing gel, run at $100 \mathrm{~W}, 1800 \mathrm{~V}$, for 2.5 h. The gel was dried with BRL Model 583 gel dryer (Cat. No. 165-1746, Bio-Rad Lab.). The $\mathrm{X}$-ray film was exposed in darkness for 1 to $2 \mathrm{~d}$ at $-70{ }^{\circ} \mathrm{C}$.

Clusteranalysis. DNA fragments generated by AFLP analysis were scored as presence (represented by 1) and absence (represented by 0 ) for each amplified fragment, respectively. Genetic similarity was calculated based on the formula as:

$$
\mathrm{GS}_{(\mathrm{i}, \mathrm{j})}=2 \mathrm{~N}_{(\mathrm{i}, \mathrm{j})} /\left[\mathrm{N}_{(\mathrm{i})}+\mathrm{N}_{(\mathrm{j})}\right]
$$

where $\mathrm{N}_{(\mathrm{i}, \mathrm{j},}$ was the number of fragments shared between individuals, while $\mathrm{N}_{(\mathrm{i})}$ and $\mathrm{N}_{(\mathrm{j})}$ were the number of fragments in the individual $i$ and j, respectively (Nei and Li, 1979). This data matrix was used in unweighted pair-group average (UPGA) cluster analysis using STATISTICA software with Percent Agreement linkage distance (SAS, 1988).

\section{Results and Discussion}

AFLP analysis. Thirty watermelon genotypes (Table 1) were screened using 64 AFLP primer combinations. Of these, eight primer combinations (Table 2) were selected for further analysis based on the number of distinct, reproducible fragments amplified among closely related genotypes (Table 1). Among the amplified fragments recorded, $13.0 \%$ to $31.9 \%$ were polymorphic within the 28 cultivars, while $43.5 \%$ to $64.2 \%$ were polymorphic among all the 30 genotypes examined (Table 2). These results indicate that the ability to detect polymorphism in watermelon germplasm is substantially greater with AFLPs compared to isozyme (Bile et al., 1989; Navot and Zamir, 1987; Zamir et al., 1984), RAPD (Hashizume et al., 1993; Lee et al., 
1996; Levi et al., 2001), and SSR (Jarret et al., 1997) analysis. Zamir et al. (1984) found that 12 commercial cultivars of $C$. lanatus were monomorphic for 19 isozyme loci examined. Likewise, Navot and Zamir (1987) reported that only four of 26 isozyme loci examined were polymorphic among 384 accessions. Bile et al. (1989) detected no polymorphic loci among eight watermelon cultivars. Using 14 random primers to differentiate 39 accessions, Lee et al. (1996) found that several genotypes still could not be distinguished from one or more other genotypes despite the high (21\%) RAPD polymorphism detected. Levi et al. (2001) provided similar results using RAPDs. Similarly, several accessions could not be differentiated based on SSR polymorphisms (Jarret et al., 1997). In contrast, the AFLP profiles were able to distinguish among all cultivars tested. These AFLP data indicate that the genetic distances within the cultivated varieties are very narrow.

Cluster analysis. Analysis of the AFLPdata for the 30 watermelon genotypes (Table 1) produced a dendrogram of genetic relatedness with similarity coefficients (SC) ranging from 0.56 to 0.99 (Fig. 1). The genotypes could be divided into three major groups (Group I, II, and III) given a similarity level of 0.82 .

Group I contains only one genotype, 'PI 296341', a Tsamma watermelon (Citrullus lanatus var. citroides) that is resistant to $F u$ sarium race 0,1 , and 2 . This accession is native to Southern Africa and is characterized by fruit possessing interior pale, firm flesh. This might have been predicted since Citrullus lanatus var. citroides has been proposed as the wild progenitor of the cultivated forms of watermelon (Bates and Robinson, 1995; Martyn and Netzer, 1991; Xuyong et al., 2000).

Group II comprises one genotype, "Egun," which is the Egusi watermelon (Citrullus lanatus var. lanatus/egusi) of West African origin (Burkill, 1985; Oyulu, 1977). Our results suggest that there is a strong genetic similarity $(\mathrm{SC}=0.720)$ between the Egusi type and the cultivated ones (Fig. 1). Therefore, Egusi types are likely related to the elite cultivars.

Group III comprises 28 cultivars (Citrullus lanatus var. lanatus) originated from East Asia and the United States. The high SC (0.82-0.99) among these accessions indicates that they have very close relationships despite diverse geographic origins. Because their pedigree relationships are conglutinate with their cluster groupings, these accessions could be further divided into three subgroups: A, B, and C. Subgroup A includes five genotypes representing classical American ecotypes and, predictably, 'Zhengkang-F' and 'Xinong-F', both of which originated from "Sugarlee" $(\mathrm{SC}=0.99)$. Nine cultivars clustered into Subgroup B have complex pedigrees and originate from the United States and East Asia as the result of selection and breeding. For instance, 'Zhengkang\#3' is the $F_{1}$ hybrid of female parent 'ZhengkangF' and male parent 'Zhengkang-M', both of which have complex ecotypic originations (Table 1). 'Sugar baby', although originating from the United States, has classical fruit characteristics typical of East Asian ecotypes.

Table 1. Watermelon [Citrullus lanatus (Thunb.) Mansf.] genotypes selected for AFLP analysis.

\begin{tabular}{llll}
\hline No. & Genotype name & \multicolumn{1}{c}{ Pedigree relationship comments } & Geographic origin \\
\hline 1 & Jingxin-F & Female parent of 'Jingxin\#1' & Japan \\
2 & 97103 & Male parent of 'Jingxin\#1' & Japan \\
3 & Jingxin\#1 & F hybrid of 'Jingxin\#1' and '97103' & Japan \\
4 & C16 & Hybrid cultivar & Japan \\
5 & Zhengza-F & Female parent of 'Zhengza\#5' & Japan \\
6 & Zhengza-M & Male parent of 'Zhengza\#5' & Japan \\
7 & Zhengza\#5 & F hybrid of 'Zhengza-F' and 'Zhengza-M' & Japan \\
8 & Zhengkang-F & Female parent of 'Zhengkang\#3'; & China \\
& & Derived from American cultivar 'sugarlee' & \\
9 & Zhengkang-M & Male parent of 'Zhengkang\#3' & Japan \\
10 & Zhengkang\#3 & F hybrid of 'Zhengkang-F' and 'Zhengkang-M' & Complex \\
11 & Heimi & Tetraploid reduplicated from 'sugar Baby' & USA \\
12 & Crimson & Hybrid cultivar & USA \\
13 & Heimi\#2 & F hybrid & China \\
14 & Xinong-F & Female parent of 'Xinong\#8' & China \\
& & Derived from American cultivar 'sugarlee' & \\
15 & Xinong-M & Male parent of 'Xinong\#8' & Japan \\
16 & Xinong\#8 & F hybrid of 'Xinong-F' and 'Xinong-M' & Complex \\
17 & T21 & Tetraploid reduplicated from 'Jingxin-F' & Japan \\
18 & T34 & Hybrid cultivar & Japan \\
19 & Huapi & Tetraploid & China \\
20 & Zhongyu\#10 & Hybrid cultivar & China \\
21 & Q201 & Hybrid with pedigree origin of '97103' & China \\
22 & Calhoun Gray & Hybrid cultivar & USA \\
23 & Sugar Baby & Hybrid cultivar & USA \\
24 & O-102 & Sister line of 'N-102' & China \\
25 & N-102 & Sister line of 'O-102' & China \\
26 & AU-Sweet Scarlet & Hybrid cultivar & USA \\
27 & $2 K-164$ & Hybrid cultivar & USA \\
28 & 2K-52 & Hybrid cultivar & USA \\
29 & PI296341 & Tsamma melon & South Africa \\
30 & Egun & Egusi melon & West Africa \\
\hline & & &
\end{tabular}

Table 2. Average number of AFLP fragments obtained from eight selected primer combinations and the polymorphism percentage within (cultivars) and among (all the 30 genotypes) accessions examined.

\begin{tabular}{lccc}
\hline $\begin{array}{l}\text { Selected AFLP } \\
\text { primer combination }\end{array}$ & $\begin{array}{c}\text { Avg no. of AFLP } \\
\text { fragment per lane }\end{array}$ & $\begin{array}{c}\text { \% Polymorphism } \\
\text { within 28 cultivars }\end{array}$ & $\begin{array}{c}\text { \% Polymorphism } \\
\text { among 30 genotypes }\end{array}$ \\
\hline E-AT/M-CTA & 94 & $31.9 \%$ & $57.4 \%$ \\
E-AT/M-CAT & 120 & $15.8 \%$ & $44.2 \%$ \\
E-AA/M-CAA & 102 & $25.5 \%$ & $52.0 \%$ \\
E-AA/M-CAC & 92 & $13.0 \%$ & $43.5 \%$ \\
E-AC/M-CTT & 76 & $18.4 \%$ & $47.4 \%$ \\
E-AG/M-CAT & 87 & $21.8 \%$ & $43.7 \%$ \\
E-ACT/M-CTG & 53 & $22.6 \%$ & $64.2 \%$ \\
E-ACT/M-CAC & 45 & $15.5 \%$ & $51.1 \%$ \\
\hline
\end{tabular}

Thus, it is possible that 'Sugar baby' has East Asian germplasm pedigree in it. Subgroup C, comprised of 14 cultivars, almost all originated from Japanese and Chinese pedigrees, can be illustrated as East Asian ecotypes. The SC values distinctly reflect the relationships among the cultivars. For instance, since 'Zhengza-M' is the male parent of $F_{1}$ hybrid 'Zhengza\#5', the fact that 'Zhengza-M' and 'Zhengza\#5' are grouped together $(\mathrm{SC}=0.99)$ can elucidate the genetic pedigree between the $\mathrm{F}_{1}$ hybrid and parents. Similarily, since 'Q201' is the hybrid with pedigree origin of '97103', while '97103' is the male parent of 'Jingxin\#1', then the grouping of 'Jingxin\#1', 'Q201', and '97103' can possibly expound the genetic relationship between them. 'O-102' and ' $\mathrm{N}-102$ ' are the sister lines mutually originated from the same parents in breeding; it is reasonable for ' $\mathrm{O}-102$ ' and ' $\mathrm{N}$ 102 ' to be grouped together with high similarity $(\mathrm{SC}=0.99)$. In cytogenetics, the genetic materials of tetraploids are reduplicated from those of diploids. Since 'T21' is the tetraploid from diploid 'Jingxin-F', the close grouping ( $\mathrm{SC}=0.99)$ of 'T21' and 'Jingxin-F' can support the result of cytogenetics.

From the dendrogram presented here, we conclude that genetic clustering results based on AFLP data can reflect the pedigree relationships, geographic ecotypic origins, and cytogenetic results of watermelon accessions.

In conclusion, our results suggest that AFLP markers are useful for genetic diversity analysis and variety identification of watermelon. The further conversion of specific amplicons to SCAR markers could enhance the value of these markers for watermelon cultivar identification.

\section{Literature Cited}

Bailey, L.H. 1930. Three discussions in Cucurbitaceae. Genet. Herb. 2:175-186.

Bates, D.M. and R. Robinson. 1995. Cucumber, melon and watermelons: Cucumis and Citrul- 


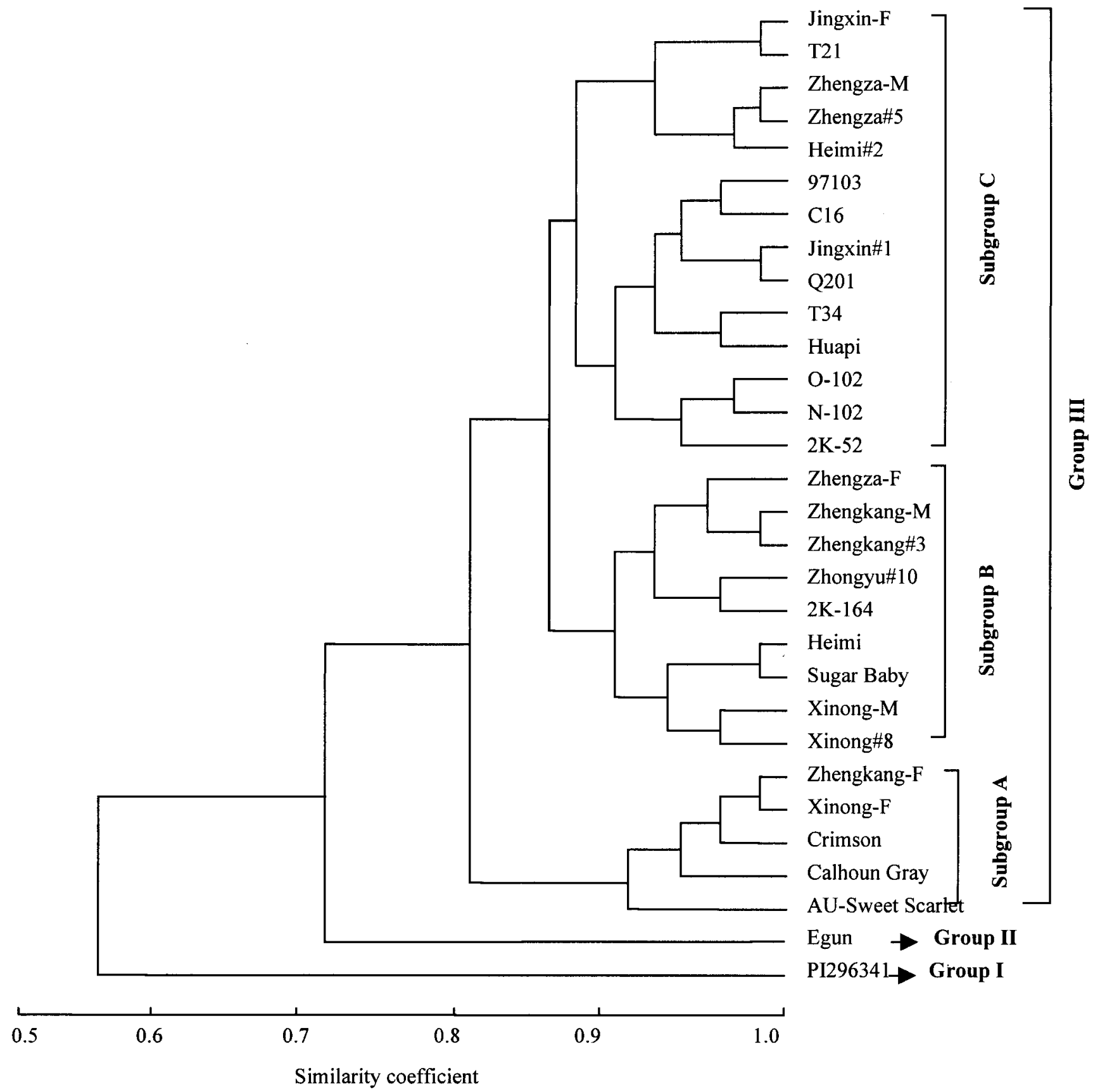

Fig.1. Genetic relationships (dendrogram) among 30 watermelon accessions based on AFLP analysis.

lus (Cucurbitaceae), p. 89-96. In: J. Smart and N.M. Simmonds (eds.). Evolution of crop plants. 2nd ed. Longman, London.

Biles, C.L., R.D. Martyn, and H.D. Wilson. 1989. Isozymes and general proteins from various watermelon cultivars and tissue types. HortScience 24:810-812.

Burkill, H.M. 1985. The useful plants of west tropical Africa. Vol. 1. 2nd ed. Royal Botanic Gardens, Kew.

Garcia-Mas, J., M. Oliver, H. Gomez-Paniagua, and M.C. de Vicente. 2000. Comparing AFLP, RAPD and RFLP markers for measuring genetic diversity in melon. Theor. Appl. Genet. 101:860-864.

Hashizume, T., T. Sato, and M. Hirai. 1993. Determination of genetic purity of hybrid seed in watermelon (Citrullus lanatus) and Lycopersicon esculentum using random amplified polymorphic
DNA (RAPD). Jpn. J. Breeding 43:367-375. Jarret, R.L., L.C. Merrick, T. Holms, J. Evans, and M.K.Aradhya. 1997. Simple sequence repeats in watermelon [Citullus lanatus (Thunb.) Matsum. \& Nakai]. Genome 40:433-441.

Jeffrey, C. 1975. Further notes on Cucurbitaceae: III. Some African taxa. Kew Bul. 30:475-493.

Lee, S.J., J.S. Shin, K.W. Park, and Y.P. Hong. 1996. Detection of genetic diversity using RAPD-PCR and sugar analysis in watermelon [Citrullus lanatus (Thunb.) Mansf.] germplasm. Theor. Appl. Genet. 92:719-725.

Levi,A., C.E. Thomas, T.C. Wehner, and X.P.Zhang. 2001. Low genetic diversity indicates the need to broaden the genetic base of cultivated watermelon. HortScience 36:1096-1101.

Li, C.Y., H.G. Zheng, M.L. Weng, J.H. Jia, T.M. Mou, H. Nguyen, and B. Wang. 2000. AFLP analysis of photoperiod sensitive genic male sterile (PGMS) rice mutant lines. J. Biotech. Sinica. 16:91-95.

Lin, J., J. Kuo, J. Ma, J.A. Saunders, H.S. Beard, M.H. MacDonald, W. Kenworthy, G.N. Ude, and B.F. Matthews. 1996. Identification of molecular markers in soybean comparing RFLP, RAPD and AFLPDNA mapping techniques. Plant Mol. Biol. Rep. 14:156-169.

Martyn, R.D. and D. Netzer. 1991. Resistance to race 0,1 and 2 of Fusarium wilt of watermelon in Citrullus sp. PI296341-FR. HortScience 26: 429-432.

Murry, H.G. and W.F. Thompson. 1980. Rapid isolation of high molecular weight plant DNA. Nucl. Acids Res. 8:4321-4325.

Navot, N. and D. Zamir. 1987. Isozyme and seed protein phylogeny of the genus Citrullus $\mathrm{C} \mathrm{Cu}$ curbitaceae). Plant Syst. Evol. 156:61-76.

Nei, M. and W.H. Li. 1979. Mathematical model for 
studying genetic variation in trems of restriction endonuclease. Proc. Natl. Acad. Sci. USA. 76: 5269-5273.

Oyulu, C. 1977. Aquantitative and qualitative study of seed types in egusi (Colocynthis citrullus L.) Trop. Sci. 19:55-62.

Pejic, I., P. Ajmone-Marsan, M. Morgante, V. Kozumplick, P. Castiglioni, G. Taramino, and M. Motto. 1998. Comparative analysis of genetic similarity among maize inbred lines detected by RFLPs, RAPDs, SSRs, and AFLPs. Theor. Appl. Genet. 97:1248-1255.

Provvidenti, R. 1994. Inheritance of a partial chlorophyll deficiency in watermelon activated by low temperatures at the seedling stage. HortScience 29:1062-1063.

Russell, J.R., J.D. Fuller, M. Macaulay, B.G. Hatz, A. Jahoor, W. Powell, and R. Waugh. 1997. Direct comparison of levels of genetic variation among barley accessions detected by RFLPs, AFLPs, SSRs, and RAPDs. Theor. Appl. Genet. 95:714-722.

SAS Institute. 1988. SAS user's guide. SAS Inst., Cary, N.C.

Sharma, S.K., M.R. Knox, and T.H.N. Ellis. 1996. AFLPanalysis of the diversity and phylogeny of Lens and its comparison with RAPD analysis. Theor. Appl. Genet. 93:751-758.

Taylor,F.W. 1989. The potential for the commercial utilization of indigenous plants in Botswana. In: G.E. Windens, J.R. Foodwin, and D.V. Fields. Plants for arid lands. Unwin Hyman, Boston.

Walters, T.W., T.S. Decker-Walters, U. Posluszny, and P.G. Kevan. 1991. Determination and interpretation of comigrating allozymes among genera of the Benincaseae (Cucurbitaceae). Syst. Bot. 16:30-40.

Wang, B., C.Y. Li, H.G. Zhang, J.M. Fu, R.C.
Yang, and H.T. Nguyen. 1999. Primary study of rice AFLP analysis-optimization of reaction condition and analysis of thermosensitive genic male sterile rice allelic mutant lines. Acta. Bot. Sinica. 41, 5:502-507.

Xu, Y., H.Y. Zhang, G.B. Kang, Y.J. Wang, and H. Chen. 2000. Studies of molecular markerassisted-selection for resistance to Fusarium wilt in watermelon (Citrullus lanatus) breeding. Acta. Genet. Sinica. 27, 2:151-157.

Zamir, D., N. Navot, and J. Rudich. 1984. Enzyme polymorphism in Citrullus lanatus and C. colocynthis in Israel and Sinai. Plant Syst. Evol. 146:163-170.

Zhang, X.P., B.B. Rhodes, and H.S. Skorupska. 1994. RAPD molucular markers in watermelon, p. 116-119. In: T. Ng. Cucurbit Genet. Coop. Rpt. No. 17. Univ. of Maryland, College Park. 